\section{Insulinothérapie Nouvelles molécules et voies d'administration}

Danilo Verge

$>$ Plusieurs études réalisées à grande échelle ont démontré la nécessité d'un contrôle strict de la glycémie chez les patients diabétiques. Des injections quotidiennes d'insuline humaine par voie sous-cutanée sont classiquement utilisées pour tenter de contrôler la glycémie. Si cette méthode permet d'obtenir une amélioration dans la régulation de la glycémie, elle ne permet pas de reproduire parfaitement le profil normal des concentrations d'insuline endogène au cours de la journée. Des avancées dans les techniques d'ingénierie des protéines ont toutefois abouti à la mise au point d'analogues de l'insuline ayant de meilleures capacités d'absorption à partir du dépôt sous-cutané, permettant une amélioration des profils insuliniques chez les patients. Dans le même temps, de nouvelles stratégies d'administration de l'insuline en sous-cutané, mais aussi par d'autres voies d'administration, ont été développées. <

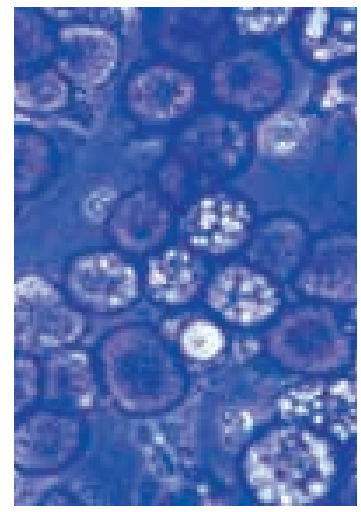

protéines a conduit au développement d'analogues de l'insuline spécialement conçus pour l'injection sous-cutanée, susceptibles d'améliorer encore le traitement des patients diabétiques.

\section{L'importance de la normoglycémie}

L'injection d'insuline exogène a pour la première fois été utilisée comme traitement du diabète en 1922, par Fred Banting et Charles Best, chez un patient présentant un diabète de type 1 , avec pour résultat une diminution de la glycémie de 4,40 à 3,20 g/l. Ce progrès, cliniquement très significatif en ce qu'il démontrait que le diabète de type 1 n'était pas nécessairement mortel, a ouvert la voie à ce qui constitue, depuis plus de 75 ans, la prise en charge du diabète, l'injection d'insuline. Au fur et à mesure des progrès de la recherche, l'insulinothérapie a eu un impact significatif sur la mortalité et la morbidité liées au diabète, et a amélioré la qualité de vie de millions de patients.

Au départ, les préparations les plus employées étaient à base d'insuline bovine ou porcine, mais l'arrivée des techniques d'ADN recombinant a permis de produire, en quantité, une insuline exogène identique à la molécule produite par le pancréas. Plus récemment, l'ingénierie des
Le profil glycémique des individus sains est caractérisé par une faible concentration plasmatique de glucose qui augmente un peu après les repas. L'allure de ce profil est assurée par une libération continue d'insuline, avec des pics de sécrétion en réponse aux apports alimentaires. Ce strict contrôle de la glycémie, qui permet de maintenir la concentration plasmatique de glucose dans une fourchette de 3,5 à $7 \mathrm{mmol} / \mathrm{I}$ [1], suggère que des concentrations sanguines plus élevées en glucose sont potentiellement dommageables.

Les résultats des études à grande échelle DCCT (diabetes control and complications trial) [2] et UKPDS (UK prospective diabetes study) [3] confortent cette hypothèse en démontrant qu'un contexte d'hyperglycémie (identifié sur la base du pourcentage d'hémoglobine glyquée HbAlc) est un facteur de risque majeur de com- 
plications microvasculaires dans les diabètes de type 1 (étude DCCT) comme dans ceux de type 2 (étude UKPDS). Dans l'étude UKPDS, par exemple, un traitement intensif réduit de $25 \%$ les paramètres microvasculaires liés au diabète. Plus encore, les deux études ont montré que toute diminution de la concentration d'HbAlc entraîne, apparemment sans effet seuil, une baisse de l'incidence des complications microvasculaires. Bien que les études montrent toutes deux que l'association entre une réduction de la glycémie et une diminution du risque cardiovasculaire est moins forte, l'analyse des données de l'UKPDS indique l'existence d'un continuum entre glycémie et risque cardiovasculaire. Par exemple, pour chaque diminution d'un point du pourcentage d'HbAlc, on observe une réduction de $25 \%$ de la mortalité toutes causes confondues et de $18 \%$ des infarctus du myocarde, qu'ils soient ou non mortels. Les résultats des études DCCT et UKPDS ont abouti à la recommandation d'utiliser des thérapies antidiabétiques intensives, avec pour objectif premier une réduction de toute hyperglycémie.

Depuis que les études DCCT et UKPDS ont été réalisées, il est devenu de plus en plus évident que la concentration postprandiale de glucose est un facteur de risque indépendant de la maladie vasculaire associée au diabète, d'où l'importance de son contrôle en termes cliniques. Par exemple, l'étude DECODE (diabetes epidemiology: collaborative analysis of diagnostic criteria in Europe), réalisée sur plus de 25000 patients, a montré que la glycémie mesurée 2 heures après une charge orale en glucose est plus étroitement associée que la glycémie à jeun à une augmentation du risque de décès [4]. De façon similaire, la Honolulu heart study trouvait une forte corrélation entre la concentration en glucose après charge et la mortalité cardiovasculaire [5], tandis que la glycémie postprandiale est retrouvée comme facteur de risque indépendant de mortalité cardiovasculaire dans la diabetes intervention study [6]. Enfin, les concentrations postprandiales de glucose sont plus étroitement corrélées avec l'HbAlc que les glycémies à jeûn, suggérant que la régulation de la glycémie postprandiale joue un rôle particulièrement important dans le maintien global de la glycémie [7].

À partir des données des études DCCT et UKPDS, et des résultats plus récents sur l'importance du contrôle de la glycémie postprandiale, l'insulinothérapie classique est devenue plus intensive. Il s'agit en particulier de reproduire au mieux les profils insuliniques quotidiens des sujets sains, et non plus seulement de diminuer les valeurs d'HbAlc. Dans cet objectif, une insulinothérapie selon un schéma basal-bolus est devenue la règle. Dans ce schéma, l'injection de préparations d'insuline à action prolongée (insuline retard) une ou deux fois par jour est complétée par une injection préprandiale d'insuline à action rapide (insuline rapide). Plusieurs avancées biotechnologiques ont permis d'optimiser le traitement basal-bolus, en particulier l'arrivée d'analogues de l'insuline. La suite de cet article est en grande partie consacrée au développement de ces analogues. Cet article fait également le point sur les nouvelles voies d'administration de ces analogues, indispensables pour optimiser leur action, et sur d'autres molécules susceptibles d'aboutir à la reproduction d'un profil glycémique normal.

\section{La nécessité de disposer d'analogues de l'insuline}

Des analogues de l'insuline ont été développés afin d'améliorer les profils pharmacocinétiques obtenus lorsque l'insuline est injectée par voie sous-cutanée. La cinétique de ces profils résulte de l'agrégation autour d'un atome de zinc de l'insuline humaine en hexamères lorsque sa concentration est élevée, un phénomène qui pourrait à l'origine avoir été prévu pour faciliter son stockage dans les vésicules des cellules $\beta$ du pancréas. En temps normal, l'exocytose de l'insuline stockée résulte en sa dilution massive, et dans sa dissociation presque immédiate en monomères; en revanche, quand l'insuline est injectée en sous-cutané, elle reste à une concentration élevée dans le dépôt et sa dissociation est, de ce fait, lente. Les hexamères (et les dimères) d'insuline pénétrant moins bien dans la paroi capillaire que le monomère biologiquement actif, l'absorption d'insuline à partir du dépôt est en partie retardée et prolongée, d'une manière qui est de plus soumise à une importante variabilité inter- comme intra-individuelle [8,9].

Ce retard dans l'absorption d'insuline humaine exogène n'est, pour diverses raisons, pas idéal pour un traitement du type basal-bolus. Tout d'abord, les injections d'insuline doivent intervenir environ 30 minutes avant les repas si l'on veut obtenir des concentrations d'insuline permettant de limiter la concentration postprandiale de glucose. Ce manque de souplesse est un inconvénient majeur pour beaucoup de sujets diabétiques et les délais d'injection se retrouvent en conséquence rarement respectés. De fait, certaines études indiquent que $75 \%$ des patients n'attendent pas 30 minutes après l'injection pour manger [10]. Par ailleurs, même si l'insuline est injectée dans un délai correct avant le repas, les concentrations sanguines en insuline restent élevées jusqu'à 8 heures après l'injection, ce qui augmente le risque de survenue d'hypoglycémie postprandiale et nécessite fréquemment un apport alimentaire 3 à 4 heures après le repas, avec pour conséquence le risque d'un gain pondéral indésirable.

\section{Le développement d'analogues de l'insuline}

L'insuline humaine est une molécule relativement simple constituée de deux chaînes d'acides aminés ( $A$ et $B$ ) reliées entre elles par deux ponts disulfures (Figure IA). La création d'analogues de l'insuline utilise en général des techniques d'ingénierie des protéines visant à modifier la séquence en acides aminés (Figure 1). J. Brange et al. ont été les premiers à publier de telles manipulations de la séquence de l'insuline 
[11]. Ils ont ainsi créé divers analogues de l'insuline qui, modifiés sur différents acides aminés, ont pour propriété commune une capacité d'auto-agrégation diminuée. Bien que ces modifications de la séquence primaire de la molécule aient pour seul objectif de modifier le profil pharmacocinétique de l'insuline, elles sont à même d'affecter la structure tridimensionnelle de la protéine, avec pour corollaire une altération possible des propriétés biologiques de l'analogue. De fait, une étude minutieuse de chaque analogue est indispensable afin de vérifier que la modification de la séquence primaire n'induit aucune propriété pharmacodynamique préjudiciable. C'est le cas notamment de l'impact possible d'une modification structurale de la protéine sur l'affinité de l'analogue pour les récepteurs de I'IGF-1 (insulin-like growth factor-1) et sur sa vitesse de dissociation des récepteurs de l'insuline. Une augmentation de l'affinité pour les récepteurs de I'IGF-I et une diminution de la dissociation des récepteurs de l'insuline ont toutes deux été mises en cause dans l'augmentation du potentiel mitogène de certains analogues, cette caractéristique ayant été préalablement associée avec le développement de tumeurs observé dans des études précliniques. Sur le nombre important d'analogues initialement étudiés, quatre seulement ont passé avec succès des tests rigoureux et sont aujourd'hui commercialisés ou en essais de phase III.

\section{Les analogues rapides de l'insuline}

Le développement d'analogues rapides de l'insuline a pour objectif d'améliorer le contrôle des oscillations de la glycémie postprandiale, c'est-à-dire de réduire son élévation. Un analogue rapide de l'insuline doit être absorbé le

Figure 1. Modifications structurales utilisées dans le développement des quatre principaux analogues de l'insuline.
A InSULINE humaine<smiles>C1CCSC1</smiles>

Gly Ile Val Glu Gln Cys Cys Thr Ser Ile Cys Ser Leu Tyr GIn Leu Glu Asn Tyr Cys Asn

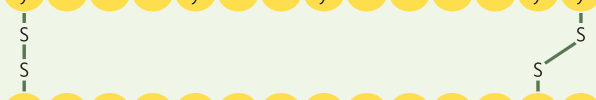

Phe Val Asn GIn His Leu Cys Gly Ser His Leu Val Glu Ala Leu Tyr Leu Val Cys Gly Glu Arg

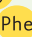

Phe

Tyr

B INSULINE LISPRO
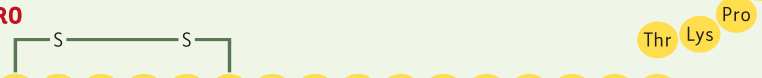

Gly Ile Val Glu GIn Cys Cys Thr Ser Ile Cys Ser Leu Tyr Gln Leu Glu Asn Tyr Cys Asn

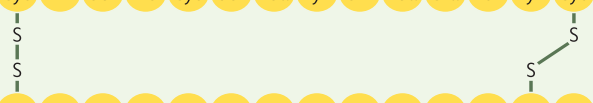

Phe Val Asn GIn His Leu Cys Gly Ser His Leu Val Glu Ala Leu Tyr Leu Val Cys Gly Glu Arg Gly

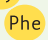

Phe

Tyr

C INSULINE ASPART
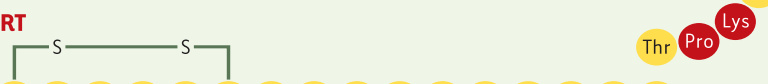

Gly Ile Val Glu Gln Cys Cys Thr Ser Ile Cys Ser Leu Tyr Gln Leu Glu Asn Tyr Cys Asn

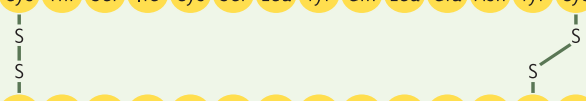

Phe Val Asn GIn His Leu Cys Gly Ser His Leu Val Glu Ala Leu Tyr Leu Val Cys Gly Glu Arg

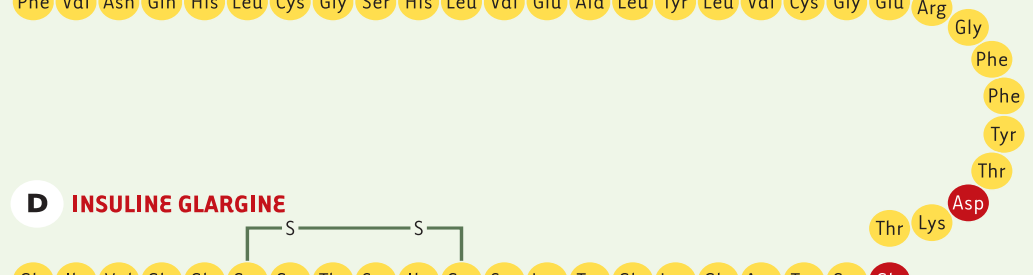

Gly Ile Val Glu Gln Cys Cys Thr Ser Ile Cys Ser Leu Tyr GIn Leu Glu Asn Tyr Cys Gly

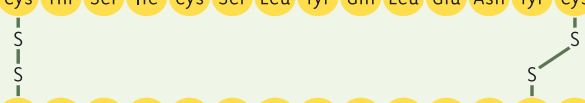

Phe Val Asn GIn His Leu Cys Gly Ser His Leu Val Glu Ala Leu Tyr Leu Val Cys Gly Glu Arg Gly

Phe

Phe

Tyr

E INSULINE DETEMIR

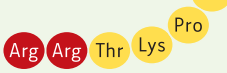

Gly Ile Val Glu Gln Cys Cys Thr Ser Ile Cys Ser Leu Tyr GIn Leu Glu Asn Tyr Cys Asn

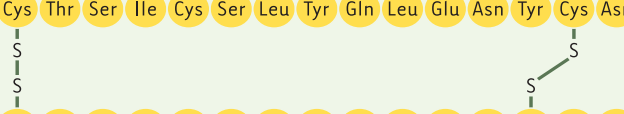

Phe Val Asn GIn His Leu Cys Gly Ser His Leu Val Glu Ala Leu Tyr Leu Val Cys Gly Glu Arg

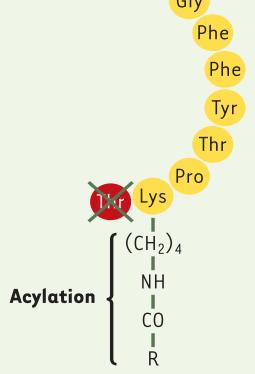


plus rapidement possible, afin d'obtenir un pic de concentration juste après l'injection, et avoir une durée d'activité assez brève par rapport à celle de l'insuline humaine exogène. Ce type de profil reproduit assez bien la situation physiologique de sécrétion de l'insuline au moment des repas, et permet d'administrer l'insuline au moment du repas, et non plus à un moment, défini strictement, avant le repas. De plus, une durée d'action plus courte permet de diminuer les grignotages visant à lutter contre l'hypoglycémie.

Puisque le principal obstacle à une absorption rapide de l'insuline humaine injectée provient de sa stabilité sous forme hexamérique lorsqu'elle est administrée en sous-cutané, le développement d'analogues rapides de l'insuline s'est principalement consacré à réduire les tendances de la molécule à s'auto-agréger [11]. Différents acides aminés situés sur la chaîne $B(B 8,9,12,16,21,23-29)$ ont été identifiés comme étant particulièrement impliqués dans ce processus, et ont donc constitué un certain nombre de cibles potentielles.

\section{L'insuline lispro}

Cet analogue est le premier à avoir été commercialisé, en 1996. Les acides aminés 28 et 29 (lysine et proline) de la chaîne $B$ ont été interchangés (Figure IB), avec pour conséquence une élimination du contact existant entre monomères au niveau des acides aminés B28 et B23, contact important pour la formation de dimères. Cette modification réduit également la force des deux liaisons hydrogènes entre les feuillets $\beta$, qui influence la stabilisation des dimères au sein des hexamères d'insuline. D'un point de vue biochimique, on observe un léger accroissement de la liaison de l'insuline lispro au récepteur de l'IGF-1, mais aucune modification dans la durée de sa dissociation des récepteurs de l'insuline [12]. Malgré cette augmentation de la liaison de l'analogue au récepteur de I'IGF-1, aucune conséquence clinique néfaste n'a été rapportée, et il est probable que les caractéristiques pharmacodynamiques de l'analogue diffèrent suffisamment peu de celles de l'insuline humaine pour qu'il y ait des conséquences en termes d'activité mitogénique.

Les études utilisant la technique du clamp euglycémique ont démontré un délai d'apparition de l'activité métabolique plus court pour l'insuline lispro que pour l'insuline humaine [13]. De plus, la concentration d'insuline au moment du pic, mesurée par le débit de glucose perfusé, est plus élevée et atteinte plus rapidement (environ 1 heure après l'injection) dans le cas de l'analogue que lorsque l'insuline humaine est injectée en souscutané [13]. Enfin, l'insuline lispro a une durée d'action plus courte que l'insuline humaine, 6 heures au lieu de 8 [13].

Les études cliniques réalisées avec l'insuline lispro sont en accord avec ses propriétés pharmacocinétiques et montrent que, chez les patients diabétiques de type 1 ou 2, l'analogue réduit les oscillations de la glycémie postprandiale par rapport à l'insuline humaine. Par exemple, une étude réalisée pendant 6 mois a comparé l'insuline lispro et l'insuline humaine dans le cadre d'un traitement basal-bolus: l'augmentation de la glycémie une heure après le repas était inférieure de $30 \%$ lors d'une injection d'insuline lispro juste avant le repas qu'après injection d'insuline humaine 30 minutes avant le repas $(2,6 \mathrm{mM}$ contre $3,7 \mathrm{mM}, \mathrm{p}<0,001)$. Cette différence se retrouvait lorsque d'autres mesures étaient effectuées en postprandial (augmentation de la glycémie prostprandiale inférieure de $53 \%$ dans le groupe lispro [ $<0,001] 2$ heures après le repas) [14]. En dépit de cette diminution de la glycémie postprandiale, la simple substitution de l'insuline humaine par la forme lispro dans le traitement basal-bolus a peu d'effet sur le pourcentage d'HbAlc. Cela est probablement dû au fait que durée d'action plus longue de l'insuline humaine compense la baisse des concentrations basales d'insuline (insuline interprandiale). Sans cette compensation, on observerait des concentrations plus élevées en glucose se reflétant au pourcentage d'HbAlc. À l'inverse, on a pu montrer une diminution significative du pourcentage d'HbAlc quand les concentrations basales d'insuline sont optimisées, par exemple lors de l'utilisation d'une perfusion continue d'insuline lispro en sous-cutané ou via une modification des doses ou du schéma d'injection d'insuline basale. Ainsi, pour des patients chez lesquels les bolus sont effectués avec de l'insuline lispro, le pourcentage d'HbAlc est diminué de $0,8 \%(p<0,001)$ si l'insuline basale est également ajustée [15]. II faut cependant noter qu'une augmentation de la quantité d'insuline basale (de $43 \%$ ) intervient probablement elle-même dans la diminution d'HbAlc.

Les études DCCT et UKPDS montrent toutes deux qu'un meilleur contrôle de la glycémie obtenu par une utilisation intensive d'insuline humaine exogène est associé à une augmentation du risque d'hypoglycémie, ce qui peut constituer un obstacle réel pour la mise en route d'un tel traitement chez les patients. Plusieurs études ont démontré une diminution significative des épisodes d'hypoglycémie chez des patients souffrant d'un diabète de type 1 ou 2, traités par l'insuline lispro. Par exemple, une étude menée en cross-over pendant 6 mois auprès de 200 patients environ présentant un diabète de type 1 montre que le pourcentage d'HbAlc n'est pas modifié, mais que les épisodes sévères d'hypoglycémie sont moins nombreux sous traitement par l'insuline lispro que sous insuline humaine (36 contre 58, $p<0,05)[16]$. De plus, il a été suggéré que cet impact de l'insuline lispro pourrait être encore plus marqué pour les épisodes nocturnes d'hypoglycémie. Une comparaison en cross over de 135 patients ayant un diabète de type 1 montre une fréquence plus faible d'épisodes symptomatiques d'hypoglycémie avec l'insuline lispro qu'avec l'insuline humaine (775 contre 1 156, $p<0,05$ ) [17]. De plus, les épisodes nocturnes sont jusqu'à trois fois moins fréquents dans le groupe lispro (52 contre 181, $p<0,001$ ).

$\varepsilon$ raison de la nature chronique de la maladie diabétique, la mesure de la qualité de vie des patients fait partie intégrante de l'évaluation de l'efficacité des différents types de traite- 
ments par l'insuline. Une adhésion au traitement, et donc un meilleur contrôle de la glycémie, seront ainsi plus facilement obtenus si les patients perçoivent les bénéfices de leur traitement, en particulier chez les diabétiques de type 2. L'amélioration de leur qualité de vie en raison d'une plus grande souplesse dans l'administration du traitement est un des bénéfices les plus fréquemment rapportés par les patients sous insuline lispro (voir [16], par exemple).

\section{L'insuline aspart}

Dans ce second analogue rapide de l'insuline commercialisé, la proline en position B28 a été remplacée par l'acide aspartique (Figure 1C). Cette modification a deux effets: le premier, comme pour l'insuline lispro, est d'éliminer toute interaction entre les monomères au niveau des acides aminés B23 et B28; le second est d'introduire un nouveau groupement carboxyle dans la chaîne, et donc une charge négative dans les conditions physiologiques, ce qui réduit encore la formation d'agrégats.

Ces modifications ont en revanche peu d'impact sur le potentiel mitogénique de la molécule: on obtient un profil très similaire à celui de l'insuline humaine en termes d'affinité pour les récepteurs de I'IGF-l comme en termes d'affinité et de dissociation des récepteurs de l'insuline [12]. L'affinité de l'insuline aspart pour le récepteur de l'insuline est légèrement supérieure à celle de l'insuline lispro, tandis que son affinité pour le récepteur de l'IGF-l comme son temps de dissociation du récepteur de l'insuline sont légèrement inférieurs. Ce profil n'indique donc aucune tendance à un accroissement du potentiel mitogénique de cet analogue par rapport à celui de l'insuline humaine.

L'insuline aspart et l'insuline lispro ont des profils pharmacodynamiques et pharmacocinétiques très similaires. Les études utilisant la technique du clamp euglycémique montrent que l'activité de l'insuline aspart apparaît plus rapidement que celle de l'insuline humaine, avec un pic au cours de la première heure après l'injection sous-cutanée et une concentration maximale pendant environ 1 heure [18, 19] (Figure 2). La diminution d'activité de l'insuline aspart est également plus rapide que celle de l'insuline humaine, avec un retour aux valeurs de base environ 6 heures après l'injection, contre 8 heures pour l'insuline humaine [18].

En accord avec le profil pharmacodynamique de l'insuline aspart, les données cliniques disponibles montrent que son injection immédiatement avant le repas réduit significativement les oscillations de la glycémie postprandiale, de façon plus importante que l'injection d'insuline 30 minutes avant le repas. Récemment, une comparaison directe de la capacité des formes aspart et lispro à contrôler la glycémie chez des patients diabétiques de type 1 a montré que les deux analogues entraînent une diminution de la glycémie postprandiale (rapport aspart/lispro des Cmax pour les oscillations de la glycémie postprandiale $=1,01$ ) [20]. Deux études réalisées à grande échelle [21, 22] ont également montré une diminution faible, mais statistiquement significative, du pourcentage d'HbAlc (environ 0,15\% d'HbAlc) après traitement par l'insuline aspart, par rapport à l'insuline humaine. Les concentrations basales d'insuline étaient optimisées dans cette étude; cependant, l'effet sur la proportion d'HbAlc reste significatif quand on tient compte des augmentations des concentrations basales d'insuline, ce qui suggère que la diminution d'HbAlc ne provient pas seulement de la variation des concentrations basales d'insuline [22].

Il a également été montré que l'utilisation d'insuline aspart au lieu d'insuline humaine soluble réduit le risque d'hypoglycémie: la fréquence globale d'épisodes hypoglycémiques est ainsi diminuée sous traitement par l'insuline aspart dans une étude menée pendant 4 semaines auprès de 90 hommes ayant un diabète de type 1 (20 épisodes contre 44 avec l'insuline humaine) [23]. Une étude récente, menée en double aveugle et en cross over auprès de 155 patients présentant un diabète de type 1 , ne montre aucune différence, en termes d'épisodes hypoglycé-

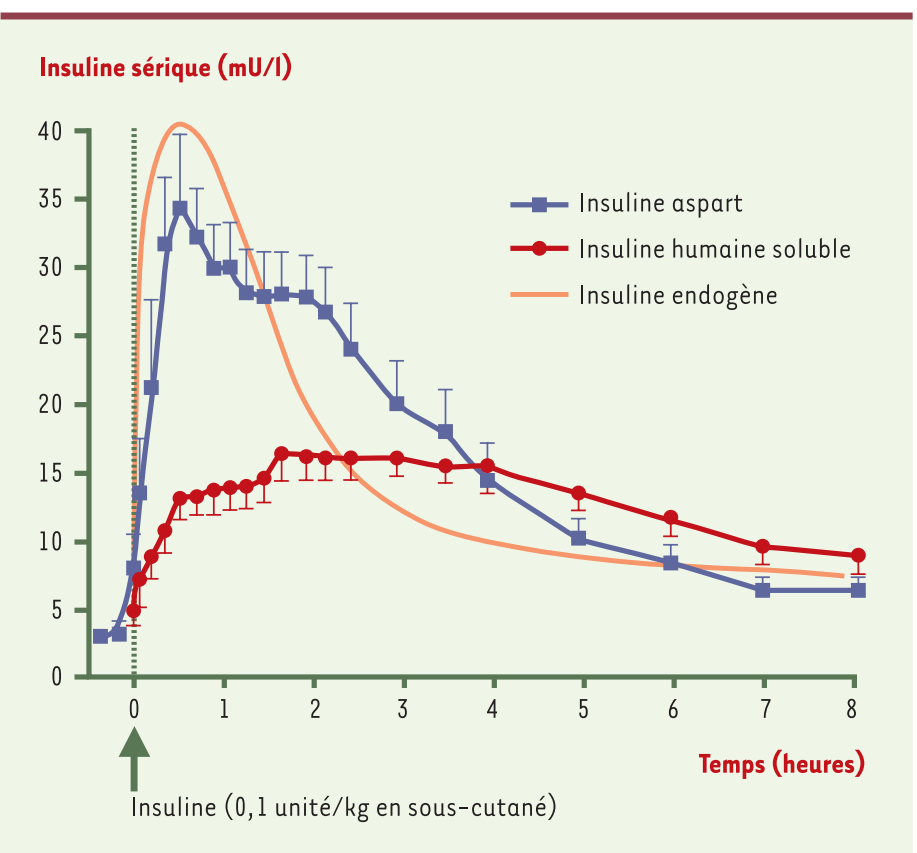

Figure 2. L'insuline aspart injectée reproduit mieux que l'insuline humaine injectée en sous-cutané le profil de l'insuline endogène après la prise d'un repas. Le profil d'insuline obtenu après l'injection d'insuline aspart présente des caractéristiques similaires à celui de l'insuline endogène. En particulier, l'action de l'insuline aspart apparaît plus rapidement, le pic d'activité est plus grand et la durée d'action est plus courte qu'avec l'insuline humaine injectée en sous-cutané [19] (d'après [45], avec l'autorisation de l'auteur). 
miques majeurs ou de pourcentage d'HbAlc, que le traitement basal-bolus s'effectue avec de l'insuline aspart ou de l'insuline humaine, mais enregistre une baisse de $72 \%$ du nombre d'événements hypoglycémiques nocturnes majeurs $(0,017$ contre 0,056 événements par mois, $p<0,005$ ) [24]. De plus, une diminution significative de la fréquence des épisodes mineurs d'hypoglycémie a également été rapportée lors d'un traitement pas l'insuline aspart (2,98 contre 3,19 événements par mois, $\mathrm{p}<0,05)$.

L'ensemble de ces résultats montre que l'utilisation d'insuline aspart au cours d'un traitement basal-bolus permet d'observer sur le plan clinique une diminution des épisodes d'hypoglycémie tout en réduisant l'hyperglycémie postprandiale, ainsi qu'un contrôle de la glycémie au moins équivalent à celui obtenu avec l'insuline humaine. L'évaluation de la qualité de vie des patients sous insuline aspart donne également des résultats très positifs. Les résultats au questionnaire de l'OMS DTSQ (diabetes treatment satisfaction questionnaire) révèlent une plus grande satisfaction des patients traités par l'insuline aspart que par l'insuline humaine $(p<0,00001)$, les différences les plus importantes étant la commodité du traitement et sa flexibilité [22].

$\varepsilon$ n conclusion, les analogues rapides de l'insuline représentent une avancée significative devant permettre de reproduire au mieux l'augmentation de l'insulinémie après les repas, typique du profil normal de l'insuline endogène. La baisse des oscillations glycémiques postprandiales après traitement par ces analogues de l'insuline devrait être bénéfique, même en l'absence d'une diminution d'HbAlc, étant donné les relations observées entre des hausses de la glycémie postprandiale et les complications diabétiques. Une meilleure perception de la qualité de vie
Insuline plasmatique

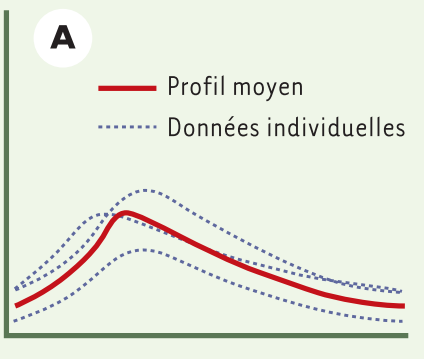

Temps
Insuline plasmatique

\section{B}

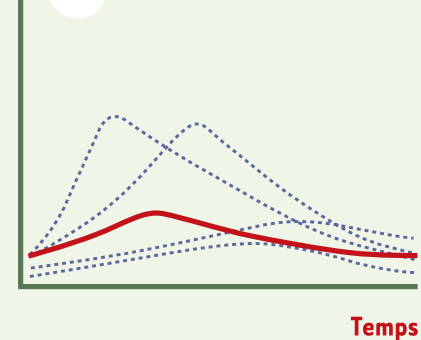

Figure 3. Profils hypothétiques des analogues de l'insuline basale. Le succès clinique d'un analogue de l'insuline ne dépend pas seulement de son profil moyen. L'injection sous-cutanée d'insuline basale est notamment sujette à une variabilité intra-individuelle significative. Le profil d'insuline basale présenté en $\boldsymbol{A}$ montre qu'une faible variabilité conduit à un profil moyen régulièrement reproductible; ce profil est ainsi vraisemblablement plus valable d'un point de vue clinique que celui montré en $B$, où une variabilité importante conduit à un profil moyen qui, bien qu'apparemment meilleur, est rarement obtenu en pratique. est également rapportée par les patients traités par ces analogues plutôt que par l'insuline humaine, en raison essentiellement d'un schéma d'administration plus pratique qui entraîne une plus grande flexibilité dans les horaires des repas. Il est clair aussi que ces analogues rapides diminue le risque d'épisodes hypoglycémiques, en particulier nocturnes. Le fait que ces analogues de l'insuline offrent une réelle alternative au traitement par l'insuline humaine est de plus en plus en reconnu, si l'on en croit l'augmentation régulière de leur prescription au cours des ans.

\section{Analogues de l'insuline basale}

Les analogues rapides de l'insuline ne peuvent pleinement remplir leur rôle qu'en présence d'insuline exogène reproduisant précisément la concentration de base normale de l'insuline endogène sécrétée. Les essais visant à recréer cette faible concentration - mais constante - à l'aide d'insuline exogène sont loins d'être satisfaisants. Les préparations d'insuline retard reposent traditionnellement sur l'addition d'agents retardants tels que le zinc (par exemple insulines lente, ultralente) ou le mélange zinc/protamine (par exemple NPH [neutral protamine Hagedorn], isophane). L'addition de ces éléments prolonge la dissociation de l'insuline, retardant ainsi l'apparition de son action (par exemple 1,5 heure pour la forme NPH et 2,5 heures pour la forme zinc lente), mais ne permet pas d'obtenir le profil dénué de pics caractéristique de l'insuline endogène de base. L'impossibilité de contrôler l'apparition de pics d'activité peut entraîner des difficultés à maintenir la glycémie dans une fourchette normale, aboutissant à des épisodes d'hypo- ou d'hyperglycémie, notamment durant la nuit.

Un autre problème de l'apport exogène d'insuline basale, peutêtre encore plus important d'un point de vue clinique, est la variabilité existant chez un même patient. Une même dose administrée à un patient peut avoir selon les jours des effets radicalement différents sur le contrôle de la glycémie (Figure 3). Bien que les recherches cliniques sur le sujet soient relativement limitées, la variabilité intra-individuelle d'action de I'insuline serait de $25 \%$ à $35 \%$ pour les insulines NPH et lente. Jusqu'à $80 \%$ de cette variabilité seraient dus aux fluctuations de l'absorption de l'insuline à partir du dépôt sous-cutané [8], le reste provenant de variations de sensibilité à l'insuline, celle-ci pouvant considérablement fluctuer au cours de la journée. Plusieurs facteurs internes (température corporelle, hormones circulantes et flux sanguin) influencent l'absorption sous-cutanée de l'insuline. De même, différents facteurs externes sont sources d'imprécision: une mise en suspension incomplète de l'insuline avant l'injection, le site d'administration, le massage de la peau avant l'injection, la pratique d'un exercice ou bien encore le tabagisme. 
Un certain nombre de stratégies ont été explorées afin d'améliorer les préparations d'insuline basale, dont l'utilisation de la pro-insuline, précurseur de l'insuline, et de l'un de ses métabolites, la Des $(64,65)$-pro-insuline humaine. Ces stratégies ont toutefois été abandonnées, principalement en raison de leur faible efficacité et de l'existence de risques cardiovasculaires possibles. Les recherches se sont alors de nouveau focalisées sur l'ingénierie des protéines, avec pour objectif, cette fois, d'obtenir de manière régulière des concentrations peu élevées d'insuline, sur une période de temps beaucoup plus longue. Le développement d'analogues de l'insuline basale efficaces d'un point de vue clinique a pris beaucoup plus de temps que celui des analogues rapides. Néanmoins, deux analogues de l'insuline basale ont franchi avec succès le stade d'une expérimentation intensive et montrent aujourd'hui un potentiel clinique prometteur.

\section{L'insuline glargine}

Cet analogue, approuvé par la FDA (food and drug administration) et I'EMEA (European agency for the evaluation of medical products) en 2000 , provient de la modification de la séquence primaire de l'insuline: l'asparagine en position 21 sur la chaîne A est remplacée par la glycine, et deux molécules d'arginine sont ajoutées sur la chaîne B (Figure ID). Ces modifications induisent une modification du point isoélectrique de la molécule, qui passe de 5,5 à 6,7. L'insuline glargine injectée en solution acide $(\mathrm{pH} 4,0)$ en sous-cutané cristallise dans cet environnement neutre, ce qui aboutit à une absorption retardée à partir du dépôt sous-cutané.

Les études montrent que l'insuline glargine présente une affinité pour le récepteur de I'IGF-l six fois plus importante que celle de l'insuline humaine et une augmentation d'un facteur 8 de la mitogénicité dans un modèle cellulaire d'ostéosarcome humain [12]. Toutefois, aucun risque carcinogène n'a été rapporté chez l'homme comme chez l'animal traités par l'insuline glargine. Il est possible que cette absence de «carcinogénicité» soit due au fait, récemment suggéré, que l'augmentation de la «mitogénicité» serait davantage liée à la vitesse de dissociation du récepteur de l'insuline qu'à la fixation au récepteur de I'IGF-1 [25]; or l'insuline glargine présente un profil de dissociation similaire à celui de l'insuline humaine.

Le profil d'activité de l'insuline glargine en fonction du temps a été évalué chez des sujets sains ou atteints de diabète de type 1 , avec la technique du clamp euglycémique et l'utilisation d'insuline glargine radiomarquée. Les résultats montrent un profil d'insuline glargine pratiquement dénué de pic, avec une activité relativement longue en comparaison de l'insuline NPH. Les études utilisant le clamp euglycémique montrent que le délai d'apparition de l'insuline glargine est de 90 minutes à 3 heures, et que son activité, pleinement atteinte en 6 heures environ, dure au moins 24 heures [25, 26]. À l'opposé, l'insuline NPH présente classiquement un pic d'activité entre 3 et
6 heures suivi d'une diminution progressive de l'activité, qui disparaît finalement 16 heures environ après l'injection. Ces résultats sont en accord avec ceux d'études de mesure de l'absorption de l'insuline utilisant des préparations radiomarquées: 3 heures environ sont nécessaires à la disparition de $25 \%$ de l'insuline NPH du dépôt sous-cutané, alors qu'il faut à peu près 9 heures avec l'insuline glargine, ce qui suggère que l'absorption de l'insuline glargine est significativement plus lente que celle de la forme NPH [27].

Si le profil cinétique moyen de l'insuline glargine est en net progrès par rapport à ceux de préparations traditionnelles d'insuline à longue durée d'action, la capacité de l'analogue à améliorer le contrôle de la glycémie semble compromise par les données montrant que son action est soumise à une variabilité intra-individuelle importante. Dans une étude utilisant la technique du clamp euglycémique, la variabilité, mesurée à I'aide du coefficient de variabilité dans les $A_{U-24}$ (aire sous la courbe des concentrations sur 24 heures), est ainsi plus élevée sur 24 heures (32\% contre 19\%) que celle de la forme NPH [28]. Cet effet est toutefois inversé lorsque l'évaluation s'effectue sur la période 12-24 heures seulement. Cette variabilité importante est susceptible d'interférer sur l'obtention régulière du profil cinétique moyen de l'insuline glargine en pratique clinique.

Une série d'études a exploré l'efficacité clinique de l'insuline glargine dans les diabètes de type 1 et 2 . Toutes ces études ont comparé l'efficacité de l'insuline glargine et de la forme NPH en évaluant divers critères de contrôle de la glycémie: pourcentage d'HbAlc, glycémie à jeun, épisodes d'hypoglycémie. Typiquement, les patients conservaient leur traitement classique (par exemple insuline de courte durée d'action au moment des repas pour les diabétiques de type 1 , agents antidiabétiques oraux pour les diabétiques de type 2 ).

Chez les diabétiques de type 1, l'insuline glargine améliorait considérablement l'équilibre entre le contrôle de la glycémie (évaluée par la glycémie à jeun) et l'incidence d'épisodes nocturnes d'hypoglycémie. Par exemple, dans une étude réalisée pendant 28 semaines, les patients recevaient soit de l'insuline glargine au moment du coucher, soit la forme NPH une ou deux fois par jour [29]. La glycémie à jeun moyenne était significativement diminuée dans le groupe glargine (de 1,3 mM, $p<0,05)$, tandis qu'aucun effet sur le pourcentage d'HbAlc n'était observé. Le nombre de patients ayant ressenti des épisodes d'hypoglycémie était également inférieur dans le groupe glargine, que ce soit globalement $(39,9 \%$ contre $49,2 \%$ pour la forme $N P H, p<0,05)$ ou plus spécifiquement la nuit $(18,2 \%$ contre 27,1\%, $p<0,05)$.

Chez les diabétiques de type 2, la capacité de l'insuline glargine d'améliorer le contrôle de la glycémie en complément du traitement par des antidiabétiques oraux est bien établie. Cependant, beaucoup de ces études n'étant pas comparatives, les avantages de cette thérapeutique par rapport à ceux 
d'autres préparations d'insuline sont moins bien évalués que dans le contexte du traitement basal-bolus. II est toutefois probable que les préparations d'insuline basale conviennent moins que les formulations prémélangées d'insuline à l'insulinothérapie additionnelle du diabète de type 2 ; en effet, les insulines basales ne sont pas indiquées pour contrôler les oscillations de la glycémie en période postprandiale, ce qui est pourtant l'aspect physiopathologique majeur du diabète de type 2.

En général, les études montrent que l'insuline glargine est bien tolérée, même si quelques patients rapportent une irritation cutanée, peut-être due à la forme acide de la préparation injectable [26]. Les explorations cliniques montrent également que l'insuline glargine influence de façon positive la qualité de vie des patients. Ces études montrent globalement une amélioration de la satisfaction liée au traitement par l'insuline glargine, en l'absence même d'une diminution démontrée de l'HbAlc. II existe également quelques preuves de la capacité de l'insuline glargine à réduire l'indicence des épisodes d'hypoglycémie perçus par les patients. En conclusion, le profil pharmacocinétique moyen de l'insuline glargine représente une avancée significative dans l'amélioration de l'apport exogène d'insuline basale, et les essais cliniques menés en comparaison de la forme NPH de l'insuline montrent que cette avancée se traduit également en termes d'amélioration clinique.

\section{Insuline detemir}

Un second analogue de l'insuline à activité prolongée, l'insuline detemir (NN304), a été commercialisé en 2004. Dans la structure de cet analogue, l'acide aminé B30, une thréonine, est éliminé, tandis que la lysine en B29 est acylée par un acide gras miristoylé à 14 atomes de carbone (Figure 1E). Comme l'insuline glargine, ces modifications permettent de prolonger le temps d'absorption à partir du dépôt sous-cutané. Cependant, à l'inverse de l'insuline glargine, cette prolongation n'est pas due à une cristallisation; l'insuline detemir se présente sous une forme soluble conservée après l'injection. L'augmentation du temps d'absorption est obtenue grâce aux chaînes d'acide gras qui augmentent la stabilité de l'hexamère, permettent la formation de di-hexamères et interfèrent avec la fixation réversible des molécules à l'albumine au niveau du tissu sous-cutané. L'ensemble de ces propriétés aboutit à un temps de demi-vie d'absorption d'environ 10 heures. Une fois dans la circulation sanguine, les monomères d'insuline detemir se fixent à nouveau de façon réversible à l'albumine (plus de $98 \%$ de fixation), ce qui prolonge encore l'action de l'insuline detemir, de façon toutefois moins importante que l'absorption lente. Heureusement, l'insuline detemir aux doses utilisées en thérapeutique n'occupe qu'une fraction des sites de fixation sur l'albumine, ce qui écarte tout danger d'interactions médicamenteuses. La séquestration de l'insuline detemir par l'albumine pourrait en revanche jouer un rôle tampon vis-à-vis de la variabilité de l'absorption. Cette caractéristique, et le fait que l'insuline detemir se dépose en solution, pourraient expliquer que les essais cliniques montrent régulièrement une diminution significative de la variabilité intra-individuelle, en termes cinétiques comme en termes dynamiques, de l'insuline detemir par rapport à la forme d'insuline NPH [30-32].

Les études utilisant la technique du clamp euglycémique montrent que le profil pharmacocinétique moyen de l'insuline detemir possède les caractéristiques nécessaires à une insuline exogène basale. L'analogue présente un délai d'apparition plus grand que la forme NPH et un pic d'activité moins prononcé [33]. L'activité maximale est obtenue, comme pour l'insuline glargine, dans les 6 heures qui suivent l'injection. Des données (non publiées) obtenues par la technique du clamp euglycémique retrouvent également cette faible variabilité d'action de l'insuline detemir. Celle-ci est également étayée par les données d'essais cliniques, qui montrent des coefficients de variation de la glycémie à jeun considérablement plus faibles que chez les patients traités par l'insuline NPH. D'autres résultats montrent que le traitement par l'insuline detemir améliore, par rapport à la forme NPH, l'équilibre entre le contrôle de la glycémie et la survenue d'épisodes nocturnes d'hypoglycémie. Des essais cliniques comparant l'analogue à la forme NPH en traitement basal-bolus suggèrent que, pour des valeurs équivalentes de glycémie à jeun, l'incidence d'hypoglycémies nocturnes serait réduite de $20 \%$ sous insuline detemir [31, 32]; ceci suggère que l'on pourrait se fixer des objectifs glycémiques plus ambitieux. Une tendance régulière, un peu surprenante, à observer une perte de poids sous traitement par l'insuline detemir par rapport au traitement par la forme NPH a également été mis en évidence dans les essais de phase III. Cette perte de poids pourrait être liée à une diminution des grignotages visant à éviter des épisodes d'hypoglycémie, la réduction de la fréquence de tels épisodes étant quant à elle liée à la baisse de variabilité intra-individuelle de l'action de l'insuline detemir, discutée plus haut. L'insuline detemir démontre donc dans les essais cliniques un fort potentiel thérapeutique en tant qu'analogue de l'insuline basale.

\section{Préparations d'insuline biphasique}

Les préparations d'insuline humaine peuvent aujourd'hui être utilisées prémélangées afin de réduire le nombre d'injections quotidiennes nécessaires. Ces préparations comprennent classiquement $30 \%$ d'insuline humaine et $70 \%$ de NPH dans une même injection, administrée avant le repas afin de fournir à la fois l'insuline basale et le bolus d'insuline ${ }^{1}$. Bien que rigides dans le rapport des doses basal-bolus, et donc peu indiquées pour le traitement du diabète de type 1 , ces préparations

1. Les mélanges contenant de l'insuline lente à longue durée d'action ont rencontré moins de succès, dans la mesure où la forme lente se modifie au cours du temps lorsqu'elle est mélangée à l'insuline humaine, en raison d'un excès de fixation d'ions zinc à l'insuline humaine. 
biphasiques peuvent être utiles chez certains patients, par exemple les diabétiques de type 2 et d'autres sujets n'ayant pas l'habitude de ce type de traitement et ne souhaitant pas rentrer dans un schéma thérapeutique aussi contraignant.

Pour optimiser l'efficacité postprandiale, les mélanges utilisant l'insuline humaine doivent être injectés environ 30 minutes avant les repas. Afin de contourner ce problème, des préparations contenant un analogue rapide de l'insuline, lispro ou aspart, ont été récemment développées. Elles contiennent l'analogue sous forme soluble dans une certaine proportion (par exemple $30 \%, 50 \%, 70 \%$ ) et sous forme protaminée. Dans des essais cliniques distincts, l'insuline biphasique aspart 30 (30\% sous forme soluble, $70 \%$ sous forme protaminée) et l'insuline biphasique lispro 25 (25\% sous forme soluble, $75 \%$ sous forme protaminée) présentaient un délai plus court d'apparition de l'activité et un meilleur contrôle de la glycémie postprandiale que l'insuline biphasique humaine 30 ( $30 \%$ insuline classique, $70 \%$ sous forme NPH) [34]. Une étude récente, comparant ces trois types de préparations biphasiques, a également montré une supériorité de l'insuline biphasique aspart 30 sur l'insuline biphasique humaine 30 et l'insuline biphasique lispro 25 à réduire la glycémie postprandiale après un repas standardisé chez des patients diabétiques de type 2 (glycémie au cours des 5 heures après le repas: $16,6 \pm 4,5$ contre $20,1 \pm 4,9$ et $18,9 \pm 60,1 \mathrm{mM} \times$ heure, respectivement; $p<0,001$ et $p<0,05[35])$.

\section{Nouveaux modes d'administration de l'insuline}

De nouveaux modes d'administration de l'insulinothérapie doivent, comme les préparations elles-mêmes, être développés. L'existence de différents dispositifs d'administration pour les patients diabétiques devrait leur permettre d'opter pour les équipements les plus appropriés à leur style de vie, conduisant en cela à une amélioration de leur adhésion au traitement et de l'évolution clinique de leur maladie. De plus, rendre l'administration d'insuline moins traumatisante et plus pratique devrait permettre de surmonter la barrière psychologique de la mise sous insuline, un problème majeur dans la prise en charge des patients ayant un diabète de type 2 .

\section{Stylos à insuline}

Aujourd'hui, l'insuline n'est classiquement plus administrée à l'aide d'une aiguille et d'une seringue. À la place, différents types de stylos injecteurs ont été développés, le premier (NovoPen, NovoNordisk) ayant été mis sur le marché en 1985. Ces stylos, qui peuvent être soit préremplis (jetables), soit réutilisables, présentent différents avantages par rapport à l'utilisation de seringues et d'aiguilles: (1) ils permettent un dosage simple, par des moyens analogiques ou numériques qui peuvent être adaptés par l'utilisateur. Par exemple, des stylos porteurs d'un grand cadran analogique ont été développés pour les personnes ayant une habileté réduite ou des difficultés d'apprentissage; (2) ces stylos sont légers et faciles à transporter; (3) on peut les adapter à toutes sortes d'aiguilles très fines, adaptées aux différents types de corpulence, ce qui améliore l'efficacité des injections et limite la douleur. Enfin, des dispositifs plus récents mémorisent les doses d'insuline administrées auparavant, tandis que d'autres équipements prévoient en plus un contrôle, bien pratique, de la glycémie.

Des études comparant les attitudes et les comportements de patients disposant de nouveaux équipements d'injection d'insuline suggèrent que des stylos bien conçus augmenteraient la perception de satisfaction des patients vis-à-vis de leur traitement, permettraient un dosage plus précis et amélioreraient l'adhésion au traitement. Par exemple, dans une étude réalisée pendant 20 semaines auprès de 21 patients ayant un diabète de type 2 récemment diagnostiqués, I'utilisation d'un stylo prérempli Novolet réduit de manière significative le pourcentage d'HbAlc par rapport au traitement utilisant des seringues conventionnelles $(7,3 \pm 1,2$ contre $7,8 \pm 1,2, p<0,02)$, ce résultat suggérant qu'il existe une meilleure adhésion au traitement avec le stylo [36]. Dans cette étude, tous les patients préféraient utiliser le NovoLet, mettant l'accent sur une administration plus rapide de l'insuline et une sélection plus facile de la dose à injecter. De plus, dans une étude comparative utilisant le FlexPen (NovoNordisk, contenant de l'insuline aspart biphasique prémélangée), $74 \%$ des patients trouvaient que ce stylo permettait une meilleure adhésion au traitement que tous les autres procédés préremplis ( $11 \%$ pour le stylo Umulin d'عlli Lilly et $0 \%$ pour l'OptiSet d'Aventis) [37].

\section{Pompes à insuline}

En alternative aux injections discontinues sous-cutanées d'insuline humaine ou d'analogues de l'insuline, la possibilité de perfuser l'insuline en continu et en sous-cutané (CSII, continuous subcutaneous insulin infusion) existe depuis quelques années et commence à rencontrer une popularité croissante. Les dispositifs pour la CSII comportent actuellement un réservoir-pompe externe et une interface de contrôle informatisée connectée à un appareil de perfusion appliqué directement sur le corps du patient (par exemple au niveau de l'abdomen). Le dispositif de perfusion délivre en continu une faible quantité d'insuline humaine en sous-cutané par l'intermédiaire d'un cathéter ou d'une aiguille, selon un schéma programmé par l'utilisateur. Théoriquement, l'insuline aspart comme l'insuline lispro (analogues rapides) sont particulièrement concernées par ces dispositifs de CSII, dans la mesure où tout changement de débit de la pompe devrait se traduire par une modification de la concentration plasmatique d'insuline en un temps minimal. Les résultats d'études cliniques menées avec des analogues rapides de l'insuline administrés par CSII vont dans ce sens. Une étude récente conduite pendant 16 semaines auprès de 146 patients souffrant d'un diabète de type 1 ayant au préalable été traités pendant 4 
semaines avec de l'insuline humaine administrée par CSII a montré que l'utilisation d'insuline aspart, lispro ou humaine en CSII aboutit à un contrôle de la glycémie équivalent et à une fréquence d'épisodes hypoglycémiques similaire [38].

Même si la CSII permet de surmonter un certain nombre d'obstacles associés à l'injection d'insuline et peut permettre d'améliorer le contrôle de la glycémie, en particulier les concentrations d'insuline basale, ce dispositif n'est pas sans poser de problèmes. Le blocage ou la panne mécanique de l'appareillage sont notamment susceptibles d'entraîner la survenue d'une acidocétose ou d'une hypoglycémie. Ces systèmes sont également plus chers, requièrent un contrôle strict, seraient plus invasifs que les injections et font courir le risque d'une infection au site de perfusion. De façon intéressante, une étude a suggéré que l'insuline aspart serait plus adaptée au dispositif par CSII que l'insuline humaine, l'insuline aspart étant moins sujette à la cristallisation dans la pompe ou dans la tubulure distale [39].

La CSII traditionnelle est un exemple de système de perfusion en circuit ouvert; l'utilisateur doit pratiquer des contrôles répétés de la glycémie afin d'établir la quantité d'insuline à perfuser. Bien que ce type de système soit utile dans le traitement du diabète, le but ultime du traitement par l'insuline est de développer un mécanisme en circuit fermé, autorégulé par rétrocontrôle, qui puisse automatiquement réagir à toute modification de la glycémie (due à un apport ou une dépense calorique) en ajustant l'administration d'insuline. Des efforts visant à recréer un «pancréas artificiel» sont en cours, notamment en termes de recherche de détecteurs potentiels de glucose. Bien que le développement d'un tel dispositif en circuit fermé soit susceptible de révolutionner l'insulinothérapie, il faut noter que même ce type de technologie sophistiquée ne pourra vraisemblablement pas reproduire parfaitement un profil insulinémique diurne normal. Ainsi, il est probable que le système fonctionnera de manière réactive (c'est-à-dire décidera d'une augmentation d'insuline en réponse à un accroissement de la glycémie) plutôt qu'anticipée, à la manière d'un pancréas sain.

\section{Administrations par des voies autres que sous-cutanées}

Tous les types de systèmes utilisant l'administration d'insuline par voie sous-cutanée se heurtent d'une part à la variabilité de l'absorption, et d'autre part au fait qu'ils peuvent constituer une barrière psychologique chez les patients entamant une insulinothérapie, en particulier chez les individus ayant une phobie des piqûres. Pour ces raisons, de nouvelles voies d'administration sont en cours de développement, l'objectif étant d'améliorer à la fois la satisfaction du patient et l'efficacité clinique de l'insuline exogène.

\section{Dispositifs en circuit ouvert implantables}

Dans ces systèmes aujourd'hui en essai clinique, la pompe à insuline est implantée sous la peau et connectée à un cathéter qui délivre l'insuline classiquement par voie intrapéritonéale (une administration intraveineuse peut aussi être employée [40]). La programmation de l'appareil est assurée par le biais d'un appareil de télémesure et la pompe est remplie de façon transcutanée toutes les 4 à 12 semaines, en fonction de la dose journalière d'insuline; ce dispositif est plus pratique pour le patient qui a moins besoin d'intervenir sur le système. Des études ont montré que ces dispositifs permettent d'obtenir un contrôle de la glycémie au moins aussi satisfaisant que les systèmes traditionnels d'injection d'insuline en sous-cutané. Une plus faible variabilité et une meilleure qualité de vie ont également été rapportées. Une amélioration du mécanisme des pompes reste néanmoins nécessaire, des essais cliniques ayant révélé un taux assez élevé de délivrance insuffisante d'insuline, résultant typiquement d'une obstruction du cathéter. Des réactions inflammatoires localisées ont également été rapportées. À condition de résoudre ces problèmes, les dispositifs de pompes implantables devraient probablement être une réussite chez certains patients, malgré leur coût financier supplémentaire. De plus, ces systèmes implantables pourraient à terme être couplés à un mécanisme de mesure de la glycémie afin de créer un système implantable en circuit fermé.

\section{Dispositifs d'administration par inhalation}

La possibilité d'administrer l'insuline par voie pulmonaire a pour la première fois été suggérée en 1925 et a été étudiée de manière importante dans les années récentes. Pour être cliniquement viable, cette voie d'administration par inhalation doit répondre à différents critères. Premièrement, le système doit délivrer l'insuline profondément dans les poumons, afin de limiter les dépôts dans la bouche et dans le tractus respiratoire supérieur. Atteindre ce but dépend essentiellement de la taille des particules obtenues par le système aérosol, les particules les plus larges ayant tendance à précipiter dans les membranes muqueuses et donc à être avalées, les particules les plus petites étant susceptibles d'être exhalées (Figure 4). Les recherches suggèrent que la taille idéale des particules devrait se situer entre 2 et $5 \mu \mathrm{m}[41,42]$. Deuxièmement, le système doit permettre de délivrer de façon reproductible une quantité suffisante d'insuline, en contournant les obstacles liés aux variations inter- et intra-individuelles dans la manière de respirer. Des dispositifs tels que AERx (NovoNordisk/Aradigm) et Exubera (Pfizer/Aventis) semblent répondre à ces critères; les essais cliniques préliminaires à leur commercialisation sont actuellement sur le point d'être achevés. Exubera est un système mécanique utilisant de l'insuline en poudre activée par un facteur déclenchant la formation d'un nuage d'insuline dans le compartiment principal du système d'administration. Une inspiration profonde permet alors d'inhaler l'insuline au niveau des poumons, où elle pénètre dans la circulation sanguine à travers la paroi alvéolaire. À l'inverse, le Diabetes management system AERx est un dispositif électronique utilisant de l'insu- 
line en solution. Ce dispositif contient des systèmes permettant un ajustement de l'amplitude respiratoire et un contrôle de facteurs environnementaux, tels que la température, afin d'assurer une inhalation de quantités précises d'insuline. L'AERx permet également, mieux que l'Exubera actuellement, une graduation plus fine des doses d'insuline délivrées.

Des études ont montré que l'administration d'insuline par ce type de systèmes permet de réduire le délai d'apparition de l'activité, le pic étant atteint 5 à 60 minutes après l'inhalation, l'activité persistant 5 à 9 heures selon la dose. Ce type de profil devrait rendre l'insuline inhalée particulièrement indiquée pour une utilisation en préprandial. De fait, dans une étude récente menée auprès de patients souffrant d'un diabète de type 2 , comparant l'administration d'insuline en préprandial soit en sous-cutané, soit par inhalation (AERx), conjointement avec une injection d'insuline NPH au coucher, on retrouvait dans les deux groupes une diminution similaire d'HbAlc au bout de 12 semaines $(0,8 \%$ et $0,7 \%$ pour l'AERx et l'insuline humaine, respectivement [43]). La glycémie à jeun était significativement plus faible dans le groupe «AERx» par rapport au groupe «sous-cutané» $(8,9$ contre $10,8 \mathrm{mM}$, respectivement, $p=0,01$ ), sans que soit observée une augmentation du risque d'hypoglycémie. En fait, le nombre total d'événements d'hypoglycémie était même plus faible dans le groupe «AERx» $(n=151)$ que dans le groupe «sous-cutané» $(n=211)$, sans toutefois que cette différence soit significative. La thérapie par l'insuline inhalée était bien tolérée, et beaucoup de patients ont choisi de la continuer à l'issue des essais. Ce type de dispositifs ne convient toutefois pas à tous les patients, notamment aux fumeurs.

\section{Administration par voie orale}

Le fait de pouvoir délivrer de l'insuline par voie orale, particulièrement sous forme de comprimé, est particulièrement séduisant, non seulement par son côté pratique, mais aussi parce que l'insuline ingérée doit passer directement par le foie, ce qui devrait la rendre plus «physiologique» (physiologiquement, l'insuline est sécrétée dans la veine porte). L'administration orale d'insuline rencontre hélas de nombreux problèmes liés à sa protélolyse dans l'estomac et à sa faible absorption directe en raison d'un défaut de systèmes sélectifs de transport de l'insuline dans la paroi intestinale. La variabilité dans la dégradation de la capsule enrobant l'insuline constitue également un obstacle. Cela explique que les efforts de développement d'une insuline administrable par voie orale aient jusqu'ici échoué. Une insuline sous forme de nébulisateur, destinée à être absorbée à travers la muqueuse buccale, pourrait être prometteuse, mais seulement si l'on utilise des agents facilitants tels que la lécithine de soja et le propanediol $[41,44]$.

\section{Administration par voie nasale}

Comme les autres modes d'administration esquissés ci-dessus, la voie nasale est attractive en raison de son côté pratique. Les recherches en la matière n'ont hélas pas rencontré de succès, les études montrant que l'administration par voie nasale ne permettaient que faiblement d'améliorer la glycémie postprandiale. Cet échec à développer un dispositif efficace a été attribué à différentes raisons. La faible surface de la muqueuse nasale, notamment, limite l'absorption. De plus, la biodisponibilité de l'insuline est soumise est à une variabilité intra- et inter-individuelle considérable, probablement en raison des quantités de mucus produites. Pour ces différentes raisons, les dispositifs d'administration de l'insuline par voie nasale ne sont plus en développement.

\section{Administration par voie transdermique}

Même si la peau constitue une barrière efficace contre l'environnement, différents systèmes ayant pour objectif de délivrer dans la circulation sanguine des molécules (par exemple de la nicotine) par voie transdermique ont été développés avec succès. Cependant, l'insuline est une molécule relativement grande et complexe, pour laquelle une administration par voie transdermique est peu évidente. En dépit de cela, un certain nombre de produits, aujourd'hui en essais cliniques de phase précoce, pourraient avec un certain succès permettre, à travers la peau, de délivrer l'insuline dans la circulation sanguine.

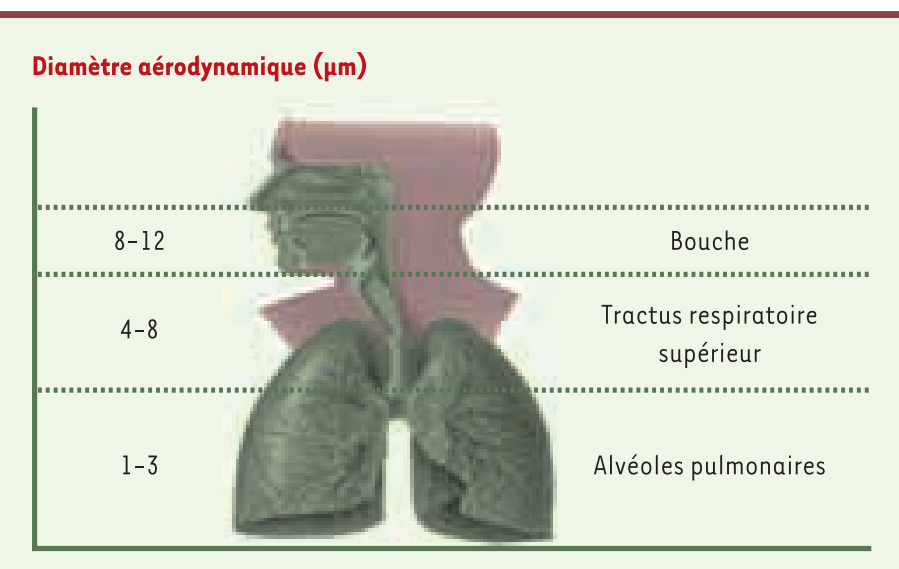

Figure 4. Importance de la taille des particules dans l'amélioration de l'absorption d'insuline par inhalation. La taille des particules est critique pour l'efficacité des préparations d'insuline administrées par inhalation. Des particules de grande taille ont tendance à précipiter dans la bouche, tandis que des particules de taille intermédiaire le feront dans la région trachéobronchique. Des préparations telles que l'AERx produisent des particules d'environ 1 à $3 \mu \mathrm{m}$, permettant un relargage de l'insuline en profondeur dans le poumon et optimisant son passage dans la circulation sanguine à travers la paroi alvéolaire (d'après [42]). 


\section{Conclusions}

Durant les 80 dernières années, la formulation de l'insuline comme son mode d'administration ont considérablement été améliorés, permettant d'obtenir un meilleur contrôle de la glycémie chez les patients diabétiques. Le développement d'analogues rapides de l'insuline, mais aussi d'analogues de l'insuline basale, a notamment permis de diversifier l'arsenal thérapeutique disponible pour les diabétologues. Ces molécules sont actuellement utilisées dans des schémas thérapeutiques qui reproduisent de façon plus fidèle les profils dynamiques de l'insuline endogène. Des recherches complémentaires sur ces analogues, ainsi que sur leur utilisation, devraient aboutir à affiner encore ces profils et continuer d'améliorer l'insulinothérapie, avec pour résultat final une diminution des risques liés au diabète. Ces avancées sur les analogues de l'insuline ont été complétées par le développement de nouveaux dispositifs d'administration sous-cutanée tels que les stylos à insuline et la CSII (continuous subcutaneous insulin infusion). Une amélioration supplémentaire a également été obtenue grâce aux progrès significatifs réalisés dans les systèmes d'administration de l'insuline par voie pulmonaire. Même si ces dispositifs nécessitent encore d'être perfectionnés, ils devraient offrir aux patients une alternative à l'administration par voie sous-cutanée dans les toutes prochaines années. $\diamond$

\section{SUMMARY}

Biotechnological and administration innovations

in insulin therapy

The importance of the intensive control of blood glucose in patients with diabetes has been well documented in several large scale studies. Attempts to attain strict glucose control when managing diabetes have traditionally utilized daily subcutaneous injections of human insulin. This strategy has offered improvements in glycaemic control but is unable to replicate fully the normal diurnal plasma profile of endogenous human insulin. Advances in protein engineering techniques have, however, resulted in the formulation of a number of insulin analogues that offer more desirable properties of absorption from the subcutaneous depot and hence improved insulin profiles in patients with diabetes. Concurrent to the development of insulin analogues, devices to deliver insulin either subcutaneously or by other routes have also advanced. These novel delivery strategies are also likely to contribute to improved glycaemic control for patients with diabetes in the future. $\diamond$

\section{RÉFÉRENCES}

1. Owens DR, Zinman B, Bolli GB. Insulins today and beyond. Lancet 2001; 358: 739-46.

2. Diabetes control and complications trial research group. The effect of intensive treatment of diabetes on the development and progression of long-term complications in insulin-dependent diabetes mellitus. N Engl J Med 1993; 329: $977-86$
3. UK Prospective diabetes study group. Intensive blood-glucose control with sulphonylureas or insulin compared with conventional treatment and risk of complications in patients with type 2 diabetes (UKPDS 33). Lancet 1998; 352: 837-53.

4. DECODE study group. Glucose tolerance and mortality: comparison of WHO and American Diabetic Association diagnostic criteria. Lancet 1999; 354: 617-21

5. Donahue RP, Abbott RD, Reed DM, Yano K. Postchallenge glucose concentration and coronary heart disease in men of Japanese ancestry (Honolulu heart program). Diabetes 1987; 36 : 689-92.

6. Hanefeld M, Fischer S, Julius U, et al. Risk factors for myocardial infarction and death in newly detected NIDDM: the diabetes intervention study, 11-year followup. Diabetologia 1996; 39 : 1577-83.

7. Avignon A, Radauceanu A, Monnier L. Nonfasting plasma glucose is a better marker of diabetic control than fasting plasma glucose in type 2 diabetes. Diabetes Care $1997 ; 20: 1822-6$.

8. Lauritzen T, Faber OK, Binder L. Variation in insulin absorption and blood glucose concentrations. Diabetologia 1979; 17: 291-5.

9. Heinemann L. Variability of insulin absorption and insulin action. Diab Technol Ther 2002; $4: 673-82$.

10. Overmann H, Heinemann L. Injection-meal interval: recommendations of diabetologists and how patients handle it. Diab Res Clin Pract 1999; 43: 137-42.

11. Brange J, Ribel U, Hansen JF, et al. Monomeric insulins obtained by protein engineering and their medical implications. Nature 1988; 333: 679-82.

12. Kurtzhals $P$, Schaffer $L$, Sorensen $A$, et al. Correlations of receptor binding and metabolic and mitogenic potencies of insulin analogs designed for clinical use. Diabetes 2000; 49: 999-1005.

13. Howey DC, Bowsher RR, Brunelle RL, Woodworth JR. ([Lys(B28), Pro(B29)])-human insulin. A rapidly absorbed analog of human insulin. Diabetes 1994; 43: 396-402.

14. Pampanelli $S$, Torlone $\varepsilon$, Ialli $C$, et al. Improved postprandial metabolic control after subcutaneous injection of a short-acting insulin analog in IDDM of short duration with residual pancreatic beta-cell function. Diabetes Care 1995; 18: 1452-9.

15. Ebeling P, Jansson PA, Smith U, et al. Strategies towards improved control during insulin lispro therapy in IDDM. Importance of basal insulin. Diabetes Care 1997; 20: $1287-9$.

16. Holleman F, Schmitt H, Rottiers R, et al. Reduced frequency of severe hypoglycaemia and coma in well-controlled IDDM patients treated with insulin lispro. The Benelux-UK Insulin Lispro study group. Diabetes Care 1997; 20: 1827-32.

17. Heller SR, Amiel SA, Mansell P. Effect of fast-acting insulin analog lispro on the risk of nocturnal hypoglycaemia during intensified insulin therapy. Diabetes Care 1999; 22: 1607-11.

18. Heinemann L, Kapitza C, Starke AAR, Heise T. Time action profile of the insulin analogue B28Asp. Diab Med 1996; 13: 683-7.

19. Home PD, Barriocanal L, Lindholm A. Comparative pharmacokinetics and pharmacodynamics of the novel rapid-acting insulin analog, insulin aspart, in healthy volunteers. Eur J Clin Pharmacol 1999; 55 : 199-203.

20. Plank J, Witter A, Brunner $G$, et al. A direct comparison of insulin aspart and insulin lispro in patients with type 1 diabetes. Diabetes Care 2002; 25 : 2053-7.

21. Raskin P, Guthrie RA, Leiter L, et al. Use of insulin aspart, a fast-acting analog, as the mealtime insulin in the management of patients with type 1 diabetes. Diabetes Care $2000 ; 23: 583-8$.

22. Home PD, Lindholm A, Riis A, et al. Insulin aspart versus human insulin in the management of long-term blood glucose control in type 1 diabetes mellitus: a randomised controlled trial. Diab Med 2000; 17: 762-70.

23. Home PD, Lindholm A, Hylleberg B, Round P. Improved glycaemic control with insulin aspart: a multicenter randomised double-blind crossover trial in type 1 diabetic patients. Diabetes Care 1998; 21 : 1904-9.

24. Colagiuri $S$, Heller $S$, Vaaler $S$, et al. Insulin aspart reduces the frequency of nocturnal hypoglycaemia in patients with type 1 diabetes. Diabetologia 2001; 44 (suppl 1): A210.

25. Bolli GB, Owens DR. Insulin glargine. Lancet $2000 ; 356$ : 443-5.

26. McKeage K, Goa KL. Insulin glargine: a review of its therapeutic use as a longacting agent for the management of type 1 and type 2 diabetes mellitus. Drugs 2001; 61: 1599-624.

27. Campbell RK, White JR, Levien T, Baker D. Insulin Glargine. Clin Ther 2001; 23: 1938-57.

28. Scholtz $H E$, van Niekerk N, Meyer BH, et al. An assessment of the variability in the pharmacodynamics (glucose lowering effect) of H0E90l compared to NPH and ultralente insulins using the euglycaemic clamp technique. Diabetologia 1999; 42 (suppl 1) : A325.

29. Ratner RE, Hirsch IB, Neifing JL, et al. Less hypoglycemia with insulin glargine in intensive insulin therapy for Type 1 diabetes. Diabetes Care 2000; 23: 639-43. 
30. Strange P, McGill J, Mazzeo M. Reduced pharmocokinetic variability of a novel, long-acting insulin analog. Diabetes 1999; 48 (suppl 1): A103.

31. Hermansen $\mathrm{K}$, Madsbad S, Perrild H, et al. Comparison of the soluble basal insulin analos insulin detemir with NFH insulin: a randomized open crossover trial in type 1 diaketic subjects on basal-bolus therapy. Diabetes Care 200; $24: 296-301$.

32. Selam JL, Skeie S, Vague $P$, et al. Prom sing results of 6 months treatment with insulin detemir in typ 1 diabetic patients. Diabetiologia 2001; 44 (suppl 1): Al5.

33. Heinemann L, Sinha K, Weyer C, et al. Time-action profile of the soluble, fatty acid acylated, long-acting insulin analogue NN304. Diab Med 1999; 16:332-8.

34. Lindholm A. New insulins in the treatment of diabetes mellitus. Best Fract Res Clin Gastroenterol 2002; 16: 475-92.

35. Hermansen K, Colombo M, Storgaard H, et al. Improved postprandial glycemic control with biphasic insulin aspart relative to biphasic human lispro and biphasic human insulin in patients with type 2 diabetes. Diabetes Care 2002; 25; 883-8.

36. Corsi A, Torre $\varepsilon$, Coronel $G$, et al. Pre-flled insulin pen in newly insulin-treated diabe:ic patients over 60 years old. Diab Nutr Metab 1997; 10: 78-81.

37. Lawton S, Berg B. Comparative evaluation of FlexPen, a new prefilled irsulin delivery system among patients and healthcare professionals. Diabetes 2001 ; 50 (suppl 1): A440.

38. Bode B, Weinstein R, Bell D, et al. Comparison of insulin aspart with buffered regulcr insulir and insulin lisp $\%$ in cortinuous subcutaneous insulin infusion: a randomized study in type 1 diabetes. Diabetes Care 2002; 25: 439-44.

39. Bode BW, Strange P. Efficacy, safety and pump compatibility of insulin aspart Lsed in continuous subcutaneous insulin infusion therapy in patients with type 1 diabe:es. Diabetes Caie $2001 ; 24: 69-72$.
40. Thompson JS, Duckworth WE. Insulin pumps and glusose regulation. Wld J Surg 2001; $25: 523-6$

41. Heinemann L, Pfützner A, Heise T. Alternative routes of administrat on as an approach to improve insulin therapy: update on dermal, oral, nasal and pulmonary insulin delivery. Curr Pharm Design 2001; 7: 1327-51.

42. By-on PR. Prediction of drug residence times in regions of the human respiratory tract following aerosol inhalation. I Pharm Sci 1986; 75 : 433-8.

43. Hermansen K, Ronnemaa T, Petersen A, Adcmson U. Intensive treatment with pulmonary insulin using AERx Insulin Diabetes Management System - a proof of concept trial in type 2 diaketic patients. Diabetes 2002; 51 (suppl 2): 194.

44. Xu HB, Hucng KX, Zhu YS, et al. Hypoglycaemic effect of a novel insulin buccal formulation on rabbits. Pharmacol Res 2002; 46: 459-67.

45. Setter SM, Corbett CF, Campbell RK, White JR. Insulin aspart: a new rapid-acting insulin anclog. Anr Pharmacother 2000; 34: 1423-31.

\section{LA RAPIDITÉ ET LA SIMPLICITÉ AVANT TOUT !}

\section{Promega vous propose ses gammes CellTiter et CytoTox : des kits pour remplacer les méthodes radioactive d'incorportion de $\left[{ }^{3} \mathrm{H}\right]$ thymidine et de relargage de $\left[{ }^{51} \mathrm{Cr}\right]$ !

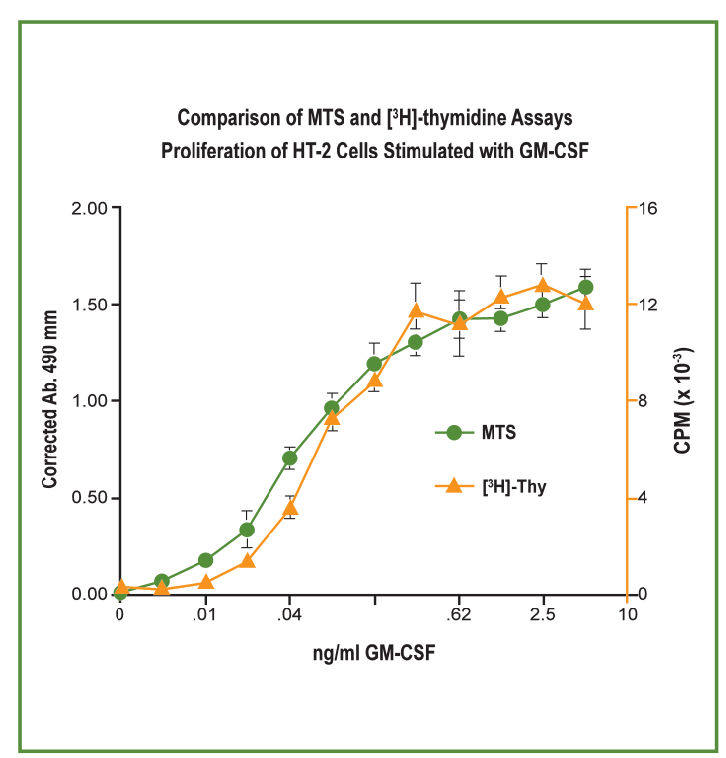 \\ Comparison of MTS and [ $\left.{ }^{3} \mathrm{H}\right]$-thymidine Assays Proliferation of HT-2 Cells Stimulated with GM-CSF \\ - une seule étape (ajouter-mesurer) \\ - ultra sensibles (jusqu'à quelques dizaines de cellules) \\ - disponibles pour tous types d'appareillages (fluorimètre, luminomètre, spectrophotomètre) \\ - non toxiques}

TIRÉS À PART

D. Verge

\section{Pour plus d'information consultez le site : www.promega.com/applications/cellprolif/ ou téléphonez au 0800488000 .}

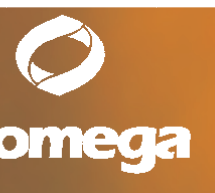

